\title{
Role of Arteriosclerosis in Development of and Recovery from Sudden Deafness and Therapeutic Effects of Lipo-PGE 1
}

\author{
Eiji Kondo ${ }^{1)}$, Ikuji Kawata ${ }^{1)}$, Tsukasa Takaishi ${ }^{2)}$, Ritsuko Abe ${ }^{3)}$, \\ Yoji Hori ${ }^{4)}$, Masakazu Goda ${ }^{5)}$, Aki Shimada ${ }^{5)}$ and Noriaki Takeda ${ }^{5)}$
}

\begin{abstract}
In order to clarify the role of arteriosclerosis in the development of and recovery from sudden deafness, we evaluated arteriosclerosis in patients with sudden deafness, using pulse wave velocity (PWV) as a marker. Arteriosclerosis was observed in $69 \%$ of the sudden deafness patients and the initial hearing level in the patients with arteriosclerosis was worse than those without arteriosclerosis. After treatment with a steroid and lipid microsphere-incorporated prostaglandin $\mathrm{E}_{1}$ (lipo- $\mathrm{PGE}_{1}$ ), the recovery rate of the patients with arteriosclerosis was significantly lower than those without arteriosclerosis. These findings suggest that arteriosclerosis is an exacerbating condition and a poor prognostic factor in patients with sudden deafness. On the other hand, PWV in the patients with arteriosclerosis who recovered tended to decrease after the treatment, suggesting that the circulation-improving effect of lipo- $\mathrm{PGE}_{1}$ may contribute to the recovery of the hearing loss related to arteriosclerosis.
\end{abstract}

Keywords : arteriosclerosis, sudden deafness, lipo- $\mathrm{PGE}_{1}$, pulse wave velocity (PWV)

\section{References}

1) Gussen R : Sudden deafness of vascular origin: a human temporal bone study. Ann Otol Rhinol Laryngol 85: 94-100, 1976.

2) Schuknecht HF, Benitez J, Beekhuis J, et al. : The pathology of sudden deafness. Laryngoscope 72: 1142-1157, 1962.

3) Saunders WH : Sudden deafness and its several treatments. Laryngoscope 82: 1206-1213, 1972.

4) Wilson WR, Byl FM and Laird N : The efficacy of steroids in the treatment of idiopathic sudden hearing loss. A double-blind clinical study. Arch Otolaryngol 106: 772-776, 1980.

5) Kubo T, Matsunaga T, Asai H, et al. : Efficacy of defibrinogenation and steroid therapies on sudden deafness. Arch Otolaryngol Head Neck Surg 114: 649-652, 1988.

6）神崎 仁, 斎藤成司 : 突発性難聴に対するプロスタグラン ディンE1の点滴静注療法. 耳鼻展望 27 補 1: 103-106, 1984.

7）荒井親雄, 長谷川元治：PWV 法. 動脈硬化症研究の進歩 11: 17-30, 1990.
8）小澤利男，増田善昭：脈波速度測定法．脈波速度．30-32 頁，メジカルビュー社，東京， 2002 .

9) Cadoni G, Scorpecci A, Cianfrone F, et al. : Serum fatty acids and cardiovascular risk factors in sudden sensorineural hearing loss: a case-control study. Ann Otol Rhinol Laryngol 119: 8288, 2010.

10）鈴木賢二, 森誠, 桝谷直司, 他：動脈硬化に関する疫 学的研究 (1) 一大動脈脈波速度と高血圧, 眼底動脈病変動 脈硬化性変化, 虚血性心電図変化との関連一. 動脈硬化 23： 715-720, 1996

11）清川重人, 原本俊一, 玉手純子, 他：PG 含有リピッドマイ クロスフェアーの動脈硬化部位へのターグティング. Drug Delivery Syst 2: 35-40, 1987.

12）笹木 収, 寺沢和貴: 突発性難聴に対するリポ $\mathrm{PGE}_{1}$ の使 用経験。新薬と臨 39: 363-368, 1990.

13) Asmar R : Arterial stiffness and pulse wave velocity. pp 17-18, Elsevier SAS, Paris, 1999.
1) Department of Otolaryngology, Anan Kyoei Hospital

2) Department of Otolaryngology, Tokushima Municipal Hospital

3) Department of Otolaryngology, Oe Kyodo Hospital

4) Department of Otolaryngology, Tokushima Prefectural Central Hospital

5) Department of Otolaryngology, University of Tokushima School of Medicine
Corresponding Author Address : Eiji Kondo

siverianj@yahoo.co.jp 
A comparison of the recovery rate in sudden deafness patients with or without arteriosclerosis.

\begin{tabular}{c|c|c|c|c}
\hline \hline & No. & Mean Age & Mean IHL & Recovery Rate \\
\hline $\begin{array}{c}\text { Arteriosclerosis }(+) \\
(\mathrm{baPWV} \geqq 1400 \mathrm{~cm} / \mathrm{s})\end{array}$ & $\begin{array}{c}20 \\
(69 \%)\end{array}$ & $\begin{array}{c}66.9 \mathrm{yr}^{*} \\
(56-78)\end{array}$ & $64.8 \mathrm{~dB}$ & $\begin{array}{c}35.0 \%^{* *}(7 / 20) \\
\text { CR 4 } \\
\text { MR 3 }\end{array}$ \\
\hline $\begin{array}{c}\text { Arteriosclerosis }(-) \\
(\mathrm{baPWV}<1400 \mathrm{~cm} / \mathrm{s})\end{array}$ & $\begin{array}{c}9 \\
\text { Total }\end{array}$ & $\begin{array}{c}47.9 \mathrm{yr}^{*} \\
(17-67)\end{array}$ & $56.5 \mathrm{~dB}$ & $\begin{array}{c}77.8 \%^{* *}(7 / 9) \\
\text { CR 6 } \\
\text { MR 1 }\end{array}$ \\
\hline 29 & $\begin{array}{c}61.0 \mathrm{yr} \\
(17-78)\end{array}$ & $62.2 \mathrm{~dB}$ & $\begin{array}{c}48.3 \% * *(14 / 29) \\
\text { CR 10 }\end{array}$ \\
& & & MR 4
\end{tabular}

$* \mathrm{P}<0.05, * * \mathrm{P}<0.05$

$\mathrm{IHL}=$ initial hearing level

$\mathrm{CR}=$ complete recovery $\quad \mathrm{MR}=$ marked recovery

The therapeutic effects of a steroid and lipo-PGE 1 and the change of PWV in the treatment of sudden deafness.

\begin{tabular}{c|c|c|c|c}
\hline \hline & & No. & baPWV decrease & baPWV increase or no change \\
\hline \multirow{2}{*}{$\begin{array}{c}\text { Arteriosclerosis }(+) \\
(\text { baPWV } \geqq 1400 \mathrm{~cm} / \mathrm{s})\end{array}$} & $\mathrm{CR}+\mathrm{MR}$ & 7 & $5(71.4 \%)$ & $2(28.6 \%)$ \\
\cline { 2 - 5 } & $\mathrm{SR}+\mathrm{NC}$ & 13 & $7(53.8 \%)$ & $6(46.2 \%)$ \\
\hline $\begin{array}{c}\text { Arteriosclerosis(-) } \\
(\mathrm{baPWV}<1400 \mathrm{~cm} / \mathrm{s})\end{array}$ & $\mathrm{CR}+\mathrm{MR}$ & 7 & $3(42.9 \%)$ & $4(57.1 \%)$ \\
\cline { 2 - 5 } & $\mathrm{SR}+\mathrm{NC}$ & 2 & $1(50.0 \%)$ & $1(50.0 \%)$ \\
\hline Total & & 29 & $16(55.2 \%)$ & $13(44.8 \%)$
\end{tabular}

$\mathrm{CR}=$ complete recovery $\quad \mathrm{MR}=$ marked recovery $\quad \mathrm{SR}=$ slight recovery $\quad \mathrm{NC}=$ no change 